\title{
Cilostazol in Acute Myocardial Infarction: New Tricks for an Old Drug?
}

\author{
Philip Adamson • Nicholas L. Cruden
}

Published online: 1 March 2014

(c) Springer International Publishing Switzerland 2014

Balancing the benefits of antiplatelet therapy with the associated bleeding risk has long been recognized as a key challenge in patients undergoing percutaneous coronary intervention. Arguably, this is most relevant in patients presenting with acute ST segment elevation myocardial infarction (STEMI) where aggressive antiplatelet and antithrombotic therapies are administered in an effort to limit myocardial injury. Progressive therapeutic developments in this area have included higher loading doses of clopidogrel [1]; alternating enthusiasm and concern with regards to glycoprotein IIb/IIIa antagonists [2, 3]; promotion of the anticoagulant agent, bivalirudin [4]; and more recently, the emergence of novel antiplatelet agents such as prasugrel and ticagrelor [5, 6]. Despite these advances, bleeding hazard remains a major problem in this population [7], particularly where patients may require early coronary artery bypass grafting [8]. The extent of academic and commercial endeavor in this area would suggest that the optimal antiplatelet treatment in patients undergoing percutaneous coronary intervention for an acute STEMI remains elusive.

An oral inhibitor of phosphodiesterase type III, cilostazol has been shown to reduce platelet aggregation, induce vascular dilatation, and limit intimal hyperplasia [9]. First approved for use in Japan in 1988, it has since been studied in a variety of vascular disorders, including peripheral arterial and cerebrovascular disease, and as an

P. Adamson · N. L. Cruden ( $\square)$

Department of Cardiology, Edinburgh Heart Centre, New Royal Infirmary of Edinburgh, Edinburgh EH16 5SA, UK

e-mail: nick.cruden@ed.ac.uk

N. L. Cruden

Centre for Cardiovascular Science, University of Edinburgh,

Edinburgh, UK adjunct to existing antiplatelet agents in patients with coronary artery disease undergoing percutaneous coronary intervention. In the current edition of the journal, Tanaka et al. [10] propose a novel indication for cilostazol, namely as a substitute for clopidogrel loading in patients presenting with STEMI.

A change in clinical practice within the authors' institution in 2009 permitted the investigators to examine clinical outcomes in patients with STEMI treated with dual antiplatelet (aspirin and clopidogrel with a $300 \mathrm{mg}$ clopidogrel loading dose) compared with those receiving triple antiplatelet therapy (aspirin, cilostazol, and clopidogrel, without a clopidogrel loading dose) [10]. The authors hypothesized that the substitution of cilostazol for clopidogrel loading in this population would reduce bleeding complications without compromising ischaemic outcomes. In fact, they found no difference in ischaemic or major bleeding outcomes between treatment groups [10]. Based on these data, the authors propose a potential role for cilostazol in the contemporary management of patients undergoing emergent percutaneous coronary intervention for STEMI. However, in reality, the single-center, retrospective, non-randomized design; the historical context; the small sample size; and lack of adjustment for confounding factors effectively limit any meaningful conclusions being drawn. Substantial further work is required before any recommendation can be made regarding the safety or efficacy of cilostazol as a substitute for clopidogrel loading in patients presenting with STEMI.

Perhaps the strongest evidence of a role for cilostazol in interventional cardiology relates to its antiproliferative effect during more prolonged use as an adjunct to conventional dual antiplatelet therapy in patients undergoing percutaneous coronary intervention [11]. A recent metaanalysis of seven randomized controlled trials comparing 
dual antiplatelet therapy (aspirin plus clopidogrel) versus triple therapy (aspirin, clopidogrel, and cilostazol), with follow-up of at least 6 months, reported a significant reduction in major adverse cardiac events with cilostazol, driven principally by lower rates of repeat revascularization, with no increase in major bleeding [12]. Intriguingly, this benefit was present irrespective of whether or not a drug-eluting stent was implanted. A post hoc analysis of pooled data from three studies would suggest that the effects of cilostazol on in-stent restenosis are directly related to the total length of stent implanted, with benefit only being observed in patients receiving a total stent length of greater than $40 \mathrm{~mm}$ [13].

Given the current global economic climate, the development of a low-cost, readily available adjunctive antiplatelet agent that improves outcomes in patients undergoing percutaneous coronary intervention is appealing. However, a number of potential barriers exist before the widespread adoption of cilostazol is likely to become reality. Almost all of the studies to date have been restricted to East Asian populations where higher rates of clopidogrel resistance have been reported [14]. The role of triple therapy with cilostazol in the setting of the newer antiplatelet agents, prasugrel and ticagrelor, is unknown. Finally, the issue of polypharmacy should not be ignored. Patients undergoing percutaneous coronary intervention are often elderly and receiving multiple medications. Adding to this burden with yet another prescription will increase the potential for drug-drug interactions and may have a negative impact on patient compliance, a strong predictor of adverse outcomes following coronary stent implantation [15]. Addressing these important issues is likely to hold the key to the future of cilostazol in the arena of interventional cardiology.

Acknowledgments Dr. Cruden currently holds a National Health Service Research Scotland Career Researcher Award.

Conflict of interest Dr. Cruden has received speakers' fees from Lilly UK. Dr. Adamson has no conflicts of interest that might be relevant to the content of this manuscript.

\section{References}

1. Mehta SR, Tanguay J-F, Eikelboom JW, Jolly SS, Joyner CD, Granger CB, et al. Double-dose versus standard-dose clopidogrel and high-dose versus low-dose aspirin in individuals undergoing percutaneous coronary intervention for acute coronary syndromes (CURRENT-OASIS 7): a randomised factorial trial. Lancet. 2010;376(9748):1233-43.
2. Neumann F-J, Kastrati A, Schmitt C, Blasini R, Hadamitzky M, Mehilli J, et al. Effect of glycoprotein IIb/IIIa receptor blockade with abciximab on clinical and angiographic restenosis rate after the placement of coronary stents following acute myocardial infarction. J Am Coll Cardiol. 2000;35(4):915-21.

3. Mehilli J, Kastrati A, Schulz S, Früngel S, Nekolla SG, Moshage $\mathrm{W}$, et al. Abciximab in patients with acute ST-segment-elevation myocardial infarction undergoing primary percutaneous coronary intervention after clopidogrel loading: a randomized double-blind trial. Circulation. 2009;119(14):1933-40.

4. Stone GW, Witzenbichler B, Guagliumi G, Peruga JZ, Brodie $\mathrm{BR}$, Dudek D, et al. Bivalirudin during primary PCI in acute myocardial infarction. N Engl J Med. 2008;358(21):2218-30.

5. Wiviott SD, Braunwald E, McCabe CH, Montalescot G, Ruzyllo W, Gottlieb S, et al. Prasugrel versus clopidogrel in patients with acute coronary syndromes. N Engl J Med. 2007;357(20):2001-15.

6. Wallentin L, Becker RC, Budaj A, Cannon CP, Emanuelsson H, Held C, et al. Ticagrelor versus clopidogrel in patients with acute coronary syndromes. N Engl J Med. 2009;361(11):1045-57.

7. Holmes JDR, Kereiakes DJ, Kleiman NS, Moliterno DJ, Patti G, Grines CL. Combining antiplatelet and anticoagulant therapies. J Am Coll Cardiol. 2009;54(2):95-109.

8. Cruden NLM, Morch K, Wong DR, Klinke WP, Ofiesh J, Hilton JD. Clopidogrel loading dose and bleeding outcomes in patients undergoing urgent coronary artery bypass grafting. Am Heart J. 2011;161(2):404-10.

9. Suh J-W, Lee S-P, Park K-W, Lee H-Y, Kang H-J, Koo B-K, et al. Multicenter randomized trial evaluating the efficacy of cilostazol on ischemic vascular complications after drug-eluting stent implantation for coronary heart disease: results of the CILON-T (Influence of CILostazol-based triple antiplatelet therapy ON ischemic complication after drug-eluting stenT implantation) Trial. J Am Coll Cardiol. 2011;57(3):280-9.

10. Tanaka A, Ishii H, Sakakibara M, Okumura S, Jinno Y, Okada K, et al. Temporary adjunctive cilostazol vs clopidogrel loading for ST-segment elevation acute myocardial infarction. Am J Cardiovasc Drugs. 2014. doi:10.1007/s40256-013-0059-7.

11. Friedland SN, Eisenberg MJ, Shimony A. Meta-analysis of randomized controlled trials on effect of cilostazol on restenosis rates and outcomes after percutaneous coronary intervention. Am J Cardiol. 2012;109(10):1397-404.

12. Ding XL, Xie C, Jiang B, Gao J, Zhang LL, Zhang H, et al. Efficacy and safety of adjunctive cilostazol to dual antiplatelet therapy after stent implantation: an updated meta-analysis of randomized controlled trials. J Cardiovasc Pharmacol Ther. 2013;18(3):222-8

13. Lee S-W, Lee J-Y, Ahn J-M, Park D-W, Han S, Park YK, et al. Comparison of dual versus triple antiplatelet therapy after drugeluting stent according to stent length (from the Pooled Analysis of DECLARE Trials). Am J Cardiol. 2013;112(11):1738-44.

14. Park KW, Kang SH, Park JJ, Yang HM, Kang HJ, Koo BK, et al. Adjunctive cilostazol versus double-dose clopidogrel after drugeluting stent implantation: the HOST-ASSURE randomized trial (Harmonizing Optimal Strategy for Treatment of Coronary Artery Stenosis-Safety \& Effectiveness of Drug-Eluting Stents \& Antiplatelet Regimen). JACC Cardiovasc Interv. 2013;6(9):932-42.

15. Iakovou I, Schmidt T, Bonizzoni E, Ge L, Sangiorgi GM, Stankovic G, et al. Incidence, predictors, and outcome of thrombosis after successful implantation of drug-eluting stents. JAMA. 2005;293(17):2126-30. 